\title{
Molecular States of Heavy Quark Mesons
}

\author{
Cheuk-Yin Wong \\ Physics Division, Oak Ridge National Laboratory, Oak Ridge, TN 37831, U.S.A.
}

(Dated: October 30, 2018)

\begin{abstract}
We explore molecular states of two open heavy-quark mesons $(Q \bar{q})-(q \bar{Q})$ in a quark-based model in terms of a four-body non-relativistic Hamiltonian with pairwise effective interactions. Molecular states are found in the combinations of $\left\{D, D^{*}, B, B^{*}\right\}$ with $\left\{\bar{D}, \bar{D}^{*}, \bar{B}, \bar{B}^{*}\right\}$, including a weaklybound $D \bar{D}^{*}$ state near the threshold which may be qualitatively identified as the 3872 state observed recently by the Belle Collaboration.
\end{abstract}

PACS numbers: 12.39.Jh,12.39.Pn

\section{INTRODUCTION}

The narrow $3872 \mathrm{MeV}$ state recently discovered by the Belle Collaboration [1], and subsequently confirmed by the CDFII Collaboration [2], has generated a great deal of interest. The state contains a $c \bar{c}$ pair and was observed in the exclusive decay of $B^{ \pm} \rightarrow K^{+} \pi^{+} \pi^{-} J / \psi$. Possible explanations of the state include a conventional charmonium state, hybrid state, and multi-quark molecular state [3-12]. Direct experimental search using $e^{+} e^{-}$annihilation by the BES Collaboration in the BEPC indicates that this state is unlikely to be a vector $1^{--}$state [13]. The 3872 state lies about $60 \mathrm{MeV}$ higher than the weighted average of the conventional $\Psi(1 D)$ charmonium state predicted by the Cornell potential [14] and the Buchmüller-Tye potential [15]. The identification of the 3872 state as $\Psi\left({ }^{3} D_{2}\right)$ would imply too large a splitting between $\Psi\left({ }^{3} D_{2}\right)$ and $\Psi\left({ }^{3} D_{1}\right)$, as the latter state has been identified with the 3770 state. Furthermore, the 3872 state is seen in the $\pi^{+} \pi^{-}$transition to $J / \psi$ and only an upper bound on E1 transition to the $\chi\left({ }^{3} P_{J}\right)$ state can be determined experimentally, whereas the $\Psi\left({ }^{3} D_{2}\right)$ state would have yielded a large partial E1 transition width, about 5 times greater than the width for the $\pi^{+} \pi^{-}$transition [16]. On the other hand, the 3872 state lies very near the $D^{* 0} \bar{D}^{0}$ threshold. The proximity of this state near the threshold and the difficulties with the $\Psi\left({ }^{3} D_{2}\right)$ description led the Belle Collaboration to suggest that the observed state may be a multi-quark 'molecular state' studied in previous theoretical investigations [3-11, 17-38].

We shall be interested in molecular states of the type $(Q \bar{q})-(q \bar{Q})$ where $Q$ is a charm or bottom quark and $q$ is a $u$ or $d$ quark. We shall often use the simplified nomenclature of " $M$ and $\bar{M}^{*}$ " or $M \bar{M}^{*}$ to denote mesons $M$ and $\bar{M}^{*}$ or their charge conjugate $M^{*}$ and $\bar{M}$. Molecular states formed by $D$ and $\bar{D}^{*}$ were predicted many years ago by Törnqvist [23] who suggested that open heavy-quark pairs form deuteron-like meson-meson states, 'deusons', because of the strong pion exchange interaction. In the heavy meson sector, Törnqvist estimated that one-pion exchange alone is strong enough to form deuteron-like composites of $B \bar{B}^{*}$ and $B^{*} \bar{B}^{*}$ bound by about $50 \mathrm{MeV}$, and composites of $D \bar{D}^{*}$ and $D^{*} \bar{D}^{*}$ bound by pion exchange alone are expected near the threshold. Manohar and Weise have also studied another type of multi-quark systems, the $(Q Q \bar{q} \bar{q})$ hadronic states, in the limit where the quark mass $m_{Q}$ of $Q$ goes to infinity. They noted that there is a long-range binding due to the one-pion exchange between ground state $Q \bar{q}$ mesons. They suggested that for two open bottom mesons, this long-range interaction may be sufficient to produce a weakly-bound two-meson state [24]. Ericson and Karl discussed the physics of pion exchange and the role of tensor forces in forming such hadronic molecules [25].

The pion-exchange model presents a reasonable description of the long-range attraction between $D$ and $\bar{D}^{*}$. However, as the state energy depends on the strength of the potential at short distances, the results of the pion-exchange model depends on how the long-range potential is regularized at short distances. The results may also be affected when exchanges of more mesons are included.

To describe the short-distance behavior, it is desirable to study multi-quark molecular states in a quark-based model. The phenomenological interaction between a quark and an antiquark in a single meson is reasonably well known $[14,15,39-44]$. However, there are considerable differences and uncertainties in the description of the interaction between constituents in a baryon, as it has been given in terms of one-gluon exchange (OGE) interactions involving color operators of the type $\boldsymbol{\lambda}(i) \cdot \boldsymbol{\lambda}(j)$ [26-38], or alternatively in terms of a one-boson meson exchange (OBE) interactions involving flavor operators of the type $\boldsymbol{\tau}(i) \cdot \boldsymbol{\tau}(j)$ [45-49]. Here, $\boldsymbol{\lambda}(i)$ is the generator of the $\mathrm{SU}(3)_{\text {color }}$ group for particle $i$ and $\tau(i)$ is the generator of the $\mathrm{SU}(2)_{\text {flavor }}$ group. The interquark interactions in the OGE and OBE models have very different color structures.

For the interaction between constituents of different mesons, Lipkin and Greenberg pointed out pathological problems involving the use of confining one-gluon exchange interactions in a quark-based model because they do not respect local color gauge invariance $[50,51]$. The underlying difficulty arises because the quark-based one-gluon exchange model represents a truncation of the basis states with the neglect of the dynamics of explicit gluon degrees of freedom. 
In spite of these defects, the quark-based one-gluon exchange model with the interchange of quarks has been used successfully to study the interaction of mesons at short distances [26, 40-44] when the dynamics of the gluon degrees of freedom can be neglected.

In a recent quark-based model study of molecular states using the one-gluon exchange model with constituent interchanges, Swanson found that the one-gluon exchange interaction couples $D \bar{D}^{*}$ with $\omega(J / \psi)$, but the interaction is not attractive enough to lead to a bound molecular state [11]. Swanson then studied molecular states with the mesonbased one-pion exchange model (as in Törnqvist [23]) and added the quark-based one-gluon exchange interaction with constituent-interchanges. The probability of the mixing of $\omega(J / \psi)$ with $D \bar{D}^{*}$ depends on the one-pion exchange potential cut-off parameter $\Lambda$, ranging from zero for the cut-off value of $\Lambda$ used by Törnqvist without the one-gluon exchange interaction [23] to a maximum mixing of $17 \%$ at large values of $\Lambda$. In such a treatment in which the interaction between quarks is superimposed on the one-pion exchange interaction between mesons, the position of the molecular state relative to the $D \bar{D}^{*}$ state and the degree of $\omega(J / \psi)$ mixing depends on how the long-range pion exchange potential is regularized at short distances and the result may also be affected when exchanges of more mesons are included.

We search for a description of the heavy-quark molecular states within a completely quark-based model. We note that the physical conditions appropriate for the molecular state of our interest have important effects on the interaction between the constituents in different mesons. The molecular state at the center of our attention has a binding energy of a few MeV. For such a weakly-bound molecular state, the average separation $R$ between the heavy mesons is considerably greater than the average radius of heavy quark mesons a (See Section III for more detail), as was already recognized by Close and Page, Voloshin, and Braaten and Kusunoki $[4,6,10]$. At these large distances with $R>>a$, the probability for the recombination of a quark of one meson with the antiquark of the other meson after a gluon exchange is highly suppressed as their quark-antiquark separation $R$ is much greater than their average natural meson radius $a$. When $R>>a$, far more likely after the exchange of a single gluon is the occurrence of the exchange of an additional gluon between quarks. This two-gluon emission leads to the color van der Waals interaction proportional to the inverse-power of $R$, as shown by Applequist et al., Peskin et al., Bhanot et al., Lipkin, Greenberg, and many other authors [50-55,60]. The color van der Waals interaction can be equivalently represented in terms of effective charges of quarks and antiquarks in a QED-type interaction. There is however no experimental evidence for the color van der Waals interaction of the inverse-power type between separated hadrons. As pointed out by Lipkin and Greenberg [50,51], it is necessary to include additional dynamics of gluons in such a quark-based model. Accordingly, due to the breaking of the gluonic string at large distance, the color van der Waals interaction should be screened by introducing a screening mass $\mu$, which will modify the inverse-power color van der Waals interaction at large distances. Appropriate for the peculiar physical conditions of weakly bound molecular states, the concepts of effective charges and the screening mass are incorporated into the effective interquark potential which is subsequently used to explore meson-meson molecular states.

Nucleus-nucleus molecular states have been observed previously in the collision of light nuclei near the Coulomb barrier [56-58]. Effective nucleon-nucleon interactions have been successfully applied to obtain the potential between two nuclei to study their reactions [58]. In a similar way, effective interquark potentials can be used to obtain the potential between mesons. Just as with the nucleus-nucleus potential, the meson-meson potential can be evaluated as a sum of a direct potential and a polarization potential. The direct potential arises from the interaction of a constituent of one meson with a constituent of another meson, and the polarization potential arises when one meson polarizes the other meson in its vicinity. Using Gaussian wave functions for the mesons, the direct potential and the polarization potential can be obtained analytically. The knowledge of the potential between the mesons then allows one to determine the eigenstate of the four-quark system.

Using the effective interaction, we find many weakly-bound molecular states in $(Q \bar{q})-(q \bar{Q})$ systems with charm and bottom quarks, including a weakly-bound $D^{+} D^{*-}$ state near the threshold which may be qualitatively identified as the 3872 state. For the bottom meson $(Q \bar{q})-(q \bar{Q})$ pairs, there are weakly-bound $2 \mathrm{~S}$ states near the threshold, in addition to $1 \mathrm{~S}$ states with binding energies of about $150 \mathrm{MeV}$.

Our exploratory study using effective charges and effective interactions in a non-relativistic quark model differs from those of the pion-exchange models and the multi-quark models with explicit single-gluon or single-boson exchanges. The results of molecular states obtained from different models can naturally be different. A careful comparison of these different results with experimental data will be useful to determine the importance of various mechanisms that are present in generating the molecular states.

This paper is organized as follows. In Section II, we start with the four-quark Hamiltonian and partition the Hamiltonian into an unperturbed part of two mesons and a residue interaction. The eigenvalue equation for the four-quark system is reduced to a Schrödinger equation for the relative motion of the two mesons in a meson-meson potential. In Section III, we present justifications for the introduction of the effective charges and the screening mass in the effective interquark interaction. In Section IV, we present our method for the evaluation of the molecular potential. Section V presents the results of the meson-meson potential and eigenvalues for four-quark states. We find 
molecular states in the combinations of $\left\{D, D^{*}, B, B^{*}\right\}$ with $\left\{\bar{D}, \bar{D}^{*}, \bar{B}, \bar{B}^{*}\right\}$. In Section VI, we discuss the origin of the short-distance attraction in $(Q \bar{q})-(q \bar{Q})$ systems and contrast them with the short-distance repulsion in $(Q \bar{q})-(Q \bar{q})$ systems. In Section VII we present our conclusions and discussions.

\section{HAMILTONIAN FOR THE FOUR-QUARK SYSTEM}

To study the $(Q \bar{q})-(q \bar{Q})$ system, we label constituents $Q, \bar{q}, q$, and $\bar{Q}$ as particles $1,2,3$, and 4 respectively and describe the four-quark system with a non-relativistic Hamiltonian

$$
H=\sum_{j=1}^{4} \frac{\boldsymbol{p}_{j}^{2}}{2 m_{j}}+\sum_{j=1}^{4} \sum_{k>j}^{4} V_{j k}+\sum_{j=1}^{4} m_{j},
$$

in which particle $j$ has a momentum $\boldsymbol{p}_{j}$ and a rest mass $m_{j}$. The pairwise interaction $V_{j k}\left(\boldsymbol{r}_{j k}\right)$ between particle $j$ and particle $k$ depends on the relative coordinate between them,

$$
\boldsymbol{r}_{j k}=\boldsymbol{r}_{j}-\boldsymbol{r}_{k}
$$

We introduce the two-body momentum

$$
\boldsymbol{P}_{j k}=\boldsymbol{p}_{j}+\boldsymbol{p}_{k}
$$

and the two-body internal relative momentum

$$
\boldsymbol{p}_{j k}=f_{k} \boldsymbol{p}_{j}-f_{j} \boldsymbol{p}_{k}
$$

where

$$
\begin{gathered}
f_{k}=m_{k} / m_{j k}, \\
m_{j k}=m_{j}+m_{k} .
\end{gathered}
$$

There are many ways to partition the total Hamiltonian $H$ into an unperturbed two-meson part and a residue interaction $V_{I}$. We partition the Hamiltonian so that in the lowest order the state in question can be described by a state of the unperturbed Hamiltonian. We can, for example, choose to partition $H$ into an unperturbed Hamiltonian $h_{12}+h_{34}$ of mesons $A(12)$ and $B(34)$ according to

$$
\begin{gathered}
H=\frac{\boldsymbol{P}_{12}^{2}}{2 m_{12}}+\frac{\boldsymbol{P}_{34}^{2}}{2 m_{34}}+V_{I}+h_{12}+h_{34}, \\
V_{I}=V_{14}\left(\boldsymbol{r}_{14}\right)+V_{13}\left(\boldsymbol{r}_{13}\right)+V_{23}\left(\boldsymbol{r}_{23}\right)+V_{24}\left(\boldsymbol{r}_{24}\right), \\
h_{j k}=\frac{\boldsymbol{p}_{j k}^{2}}{2 \mu_{j k}}+V_{j k}\left(\boldsymbol{r}_{j}-\boldsymbol{r}_{k}\right)+m_{j k} \quad \text { for }(j k)=A(12) \text { and } B(34),
\end{gathered}
$$

where $\mu_{j k}=m_{j} m_{k} / m_{j k}$. The eigenvalues of the Hamiltonians $h_{12}$ and $h_{34}$ can be solved separately to obtain the bound state wave functions and masses $M_{j k}(\nu)$ of mesons $A$ and $B$,

$$
h_{j k}\left|(j k)_{\nu}\right\rangle=\left[\epsilon_{j k}(\nu)+m_{j k}\right]\left|(j k)_{\nu}\right\rangle=M_{j k}(\nu)\left|(j k)_{\nu}\right\rangle .
$$

The four-body Hamiltonian becomes

$$
H=\frac{\boldsymbol{P}_{12}^{2}}{2 m_{12}}+\frac{\boldsymbol{P}_{34}^{2}}{2 m_{34}}+V_{I}+M_{12}(\nu)+M_{34}\left(\nu^{\prime}\right) .
$$

The above non-relativistic approximation is obtained from relativistic results by neglecting terms of order $\epsilon_{j k} / M_{j k}$ and higher [59]. In order to satisfy the boundary condition at large separations for which $V_{I}$ approaches zero, we need 
to include some of these higher-order terms and modify $m_{12}$ and $m_{34}$ in the above equation to $M_{12}(\nu)$ and $M_{34}\left(\nu^{\prime}\right)$ so that the Hamiltonian becomes

$$
H=\frac{\boldsymbol{P}_{12}^{2}}{2 M_{12}(\nu)}+\frac{\boldsymbol{P}_{34}^{2}}{2 M_{34}\left(\nu^{\prime}\right)}+V_{I}+M_{12}(\nu)+M_{34}\left(\nu^{\prime}\right) .
$$

The above Hamiltonian then describes properly the asymptotic behavior at large separations of the two mesons.

To solve for the eigenstates, we use the two-body meson states $\left|A_{\nu}(12) B_{\nu^{\prime}}(34)\right\rangle$ as basis states. We consider a four-quark system in which the mesons would be in the $\left|A(12)_{\nu} B(34)_{\nu^{\prime}}\right\rangle$ state if there were no residue interactions $V_{I}$. When we include the residue interactions $V_{I}$ as a perturbation, the eigenfunction of $H$ becomes

$$
\Psi\left(\boldsymbol{r}, \boldsymbol{r}_{12}, \boldsymbol{r}_{34}\right)=\psi(\boldsymbol{r})\left\{\left|A_{\nu} B_{\nu^{\prime}}\right\rangle-\sum_{\lambda, \lambda^{\prime}}^{\prime} \frac{\left|A_{\lambda} B_{\lambda^{\prime}}\right\rangle\left\langle A_{\lambda} B_{\lambda^{\prime}}\left|V_{I}\right| A_{\nu} B_{\nu^{\prime}}\right\rangle}{\epsilon\left(A_{\lambda}\right)+\epsilon\left(B_{\lambda^{\prime}}\right)-\epsilon\left(A_{\nu}\right)-\epsilon\left(B_{\nu}^{\prime}\right)}\right\},
$$

where

$$
\boldsymbol{r}=\boldsymbol{R}_{12}-\boldsymbol{R}_{34}
$$

and $\boldsymbol{R}_{j k}$ is the center-of-mass coordinate of $m_{j}$ and $m_{k}$,

$$
\boldsymbol{R}_{j k}=f_{j} \boldsymbol{r}_{j}+f_{k} \boldsymbol{r}_{k} .
$$

The spatial coordinates of the four-particle system and $\boldsymbol{r}, \boldsymbol{r}_{j k}$, and $\boldsymbol{R}_{j k}$ are shown in Fig. 1.

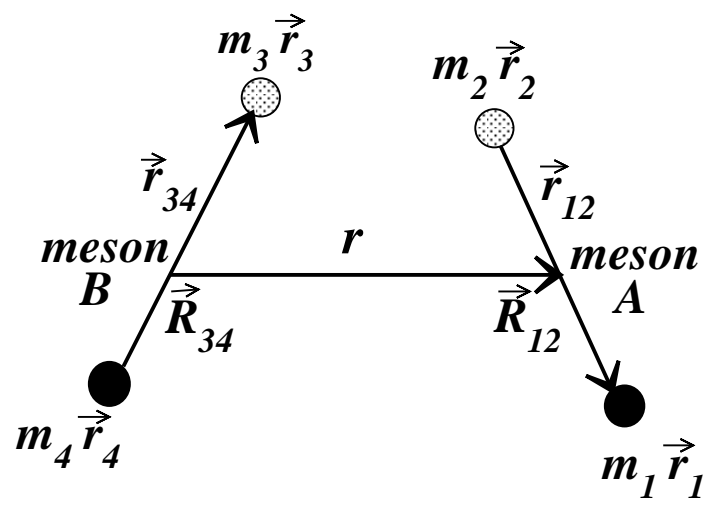

FIG. 1: The spatial coordinates of the four-particle system.

The wave function $\psi(\boldsymbol{r})$ describes the relative motion between the two mesons and the symbol $\sum_{\lambda \lambda^{\prime}}^{\prime}$ is to indicate that the sum is over all meson states except $\left|A_{\nu} B_{\nu^{\prime}}\right\rangle$. The eigenvalue equation is

$$
H \Psi\left(\boldsymbol{r}, \boldsymbol{r}_{12}, \boldsymbol{r}_{34}\right)=\left[M_{12}(\nu)+M_{34}\left(\nu^{\prime}\right)+\epsilon\right] \Psi\left(\boldsymbol{r}, \boldsymbol{r}_{12}, \boldsymbol{r}_{34}\right) .
$$

Working in the center-of-mass frame and taking the scalar product of the above equation with $\left|A_{\nu} B_{\nu^{\prime}}\right\rangle$, we obtain the Schrödinger equation for relative motion of mesons $A_{\nu}(12)$ and $B_{\nu^{\prime}}(34)$,

$$
\left\{{\frac{\boldsymbol{p}^{2}}{2 \mu}}_{A B}+V(r)\right\} \psi(\boldsymbol{r})=\epsilon \psi(\boldsymbol{r})
$$

where $\boldsymbol{p}$ is the relative momentum

$$
\boldsymbol{p}=\frac{M_{34}\left(\nu^{\prime}\right) \boldsymbol{P}_{12}-M_{12}(\nu) \boldsymbol{P}_{34}}{M_{12}(\nu)+M_{34}\left(\nu^{\prime}\right)}
$$

and $\mu_{A B}$ is the reduced mass of the two mesons

$$
\mu_{A B}=\frac{M_{12}(\nu) M_{34}\left(\nu^{\prime}\right)}{M_{12}(\nu)+M_{34}\left(\nu^{\prime}\right)} .
$$


The meson-meson potential $V(\boldsymbol{r})$ is given by

$$
V(\boldsymbol{r})=\left\langle A_{\nu} B_{\nu^{\prime}}\left|V_{I}\right| A_{\nu} B_{\nu^{\prime}}\right\rangle-\sum_{\lambda, \lambda^{\prime}}^{\prime} \frac{\left|\left\langle A_{\lambda} B_{\lambda^{\prime}}\left|V_{I}\right| A_{\nu} B_{\nu^{\prime}}\right\rangle\right|^{2}}{\epsilon\left(A_{\lambda}\right)+\epsilon\left(B_{\lambda^{\prime}}\right)-\epsilon\left(A_{\nu}\right)-\epsilon\left(B_{\nu^{\prime}}\right)} .
$$

We shall call the first term the direct potential

$$
V_{\mathrm{dir}}(\boldsymbol{r})=\left\langle A_{\nu}(12) B_{\nu^{\prime}}(34)\left|V_{I}\left(\boldsymbol{r}, \boldsymbol{r}_{12}, \boldsymbol{r}_{34}\right)\right| A_{\nu}(12) B_{\nu^{\prime}}(34)\right\rangle .
$$

It arises by the direct interaction of the quark matter densities of the projectile meson with the quark matter density of the target meson mediated by the residue interaction $V_{I}$. We shall call the second term the polarization potential

$$
V_{\mathrm{pol}}(\boldsymbol{r})=-\sum_{\lambda, \lambda^{\prime}}^{\prime} \frac{\left|\left\langle A_{\lambda}(12) B_{\lambda^{\prime}}(34)\left|V_{I}\left(\boldsymbol{r}, \boldsymbol{r}_{12}, \boldsymbol{r}_{34}\right)\right| A_{\nu}(12) B_{\nu^{\prime}}(34)\right\rangle\right|^{2}}{\epsilon\left(A_{\lambda}\right)+\epsilon\left(B_{\lambda^{\prime}}\right)-\epsilon\left(A_{\nu}\right)-\epsilon\left(B_{\nu^{\prime}}\right)} .
$$

It is always attractive and it arises from the excitation of the colliding mesons into intermediate states due to the residue interaction $V_{I}$, as in the polarization of a meson in the vicinity of another meson. When the potential $V(\boldsymbol{r})$ is determined and the eigenvalue $\epsilon$ of the Schrödinger equation [Eq. (17)] is obtained, the mass of the four-quark system will be given by $M=M_{12}(\nu)+M_{34}\left(\nu^{\prime}\right)+\epsilon$.

\section{EFFECTIVE INTERACTIONS}

We shall consider the lowest energy state for which the unperturbed mesons are in their ground states with $\nu=$ $\nu^{\prime}=0$. The energy of the four-quark system depends on the interquark interaction. The low-energy properties of isolated mesons can be described reasonably well by a quark and an antiquark interacting with a phenomenological confining one-gluon exchange interaction [14,15, 39-44]. When generalized to the multi-quark interactions involving two quark-antiquark pairs, the one-gluon exchange interaction contains a color operator of the type $\boldsymbol{F}(i) \cdot \boldsymbol{F}(j)$, where $\boldsymbol{F}(i)$ is $\boldsymbol{\lambda}(i) / 2$ for a quark and is $\left(-\boldsymbol{\lambda}^{\dagger}(i) / 2\right)$ for an antiquark. It was realized by Lipkin and Greenberg that such a quark-based one-gluon exchange model violates local color gauge invariance because of the neglect of the gluon dynamical degrees of freedom $[50,51]$. Nevertheless, the quark-based one-gluon exchange model has been used successfully to study the interaction between mesons at short distances [26, 40-44] when the gluon dynamical degrees of freedom can be neglected.

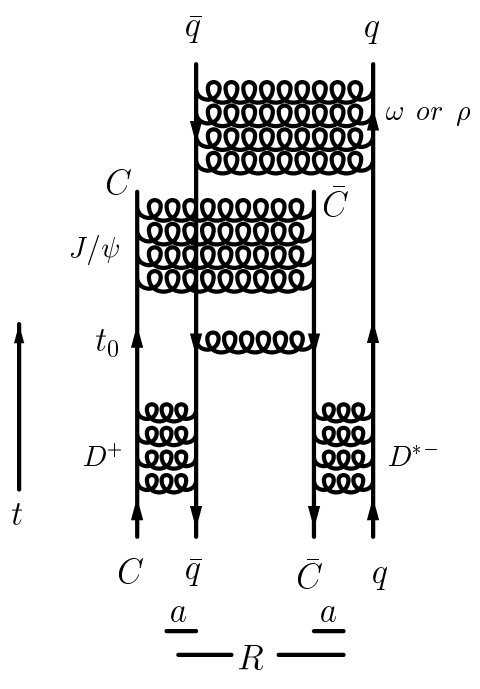

(a)

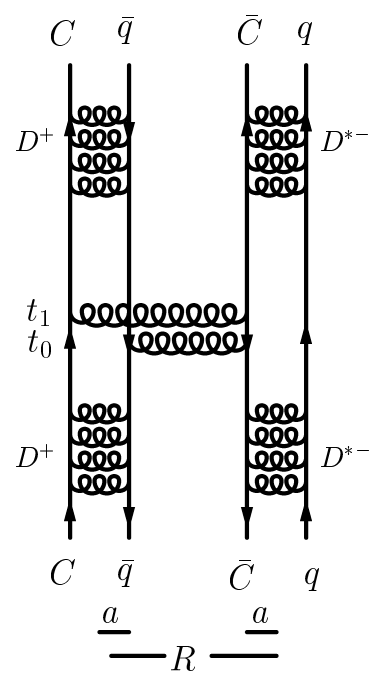

(b)

FIG. 2: Two different ways to neutralize the color after exchanging a gluon at time $t_{0}$ : $(a)$ Color can be neutralized by the $C$ interacting with the $\bar{C}$ to form a $J / \psi$, and the $q$ interacting with the $\bar{q}$ to form a $\omega$ or $\rho$. (b) Color can be alternatively neutralized by exchanging another gluon at $t_{1}$. The multi-gluon exchanges in the initial and final states represent schematically non-perturbative QCD interactions leading to bound meson states. $R$ is the separation between mesons and $a$ is the average meson radius.

When one applies the one-gluon exchange model to study the interaction between mesons, the two mesons become color octet states after a gluon is exchanged between them. The colors of the two mesons can be neutralized when a 
quark of one meson interacts with the antiquark of the other meson to form a bound color-singlet state (Fig. 2a), as described by the quark interchange model of Barnes and Swanson [40, 41]. When this quark-based model is applied to study heavy-quark molecular states, Swanson found that the one-gluon exchange interaction couples $D \bar{D}^{*}$ with $\omega(J / \psi)$, but the interaction is not attractive enough to lead to a bound molecular state [11]. Swanson then studied molecular states with the meson-based one-pion exchange model and added the quark-based one-gluon exchange interaction with constituent-interchanges. He found that the probability of the mixing of $\omega(J / \psi)$ with $D \bar{D}^{*}$ depends on the one-pion exchange potential cut-off parameter $\Lambda$, ranging from zero for the cut-off value of $\Lambda$ used by Törnqvist without the one-gluon exchange interaction [23] to a maximum mixing of $17 \%$ at large values of $\Lambda$.

To study heavy-quark molecular states within a completely quark-based model, we note that the physical conditions associated with the molecular state of our interest have important effects on the interquark interaction. We consider, for example, the molecular state of $D^{+} D^{*-}$ to be a possible description of the $3872 \mathrm{MeV}$ state. It is then a weaklybound molecular state with a binding energy $\epsilon$ given by $\epsilon=\left[3.872 \mathrm{GeV}-M(D+)-M\left(D^{*-}\right)\right]=7.4 \mathrm{MeV}$. The average separation $R$ between $D^{+}$and $D^{*-}$ is of the order of $R \sim \hbar / \sqrt{2 m_{\text {red }} \epsilon}$ where $m_{\text {red }}$ is the reduced mass, $M\left(D^{+}\right) M\left(D^{*-}\right) /\left(M(D+)+M\left(D^{*-}\right)\right)$. For the binding energy of $7.4 \mathrm{MeV}$, we can estimate the average separation $R$ between $D^{+}$and $D^{*-}$ to be about $1.6 \mathrm{fm}$. This separation $R$ is much greater than the average radius $a$ of $D^{+}$and $D^{*-}$, whose root-mean-square radius is about $0.3 \mathrm{fm}$ as given by half of the calculated root-mean-square quark-antiquark separation listed in the Appendix of [42]. Our molecular state is therefore characterized by an average meson-meson separation $R$ much greater than the average radius $a$ of the mesons. This peculiar feature of $R>>a$ has already been recognized by Close and Page, Voloshin, and Braaten and Kusunoki [4, 6, 10].

After a gluon is exchange between $D^{+}$and $D^{*-}$ at $t_{0}$, the $C$ and $\bar{C}$ are separated by a distance of the order of the meson-meson separation $R$, as depicted in Fig. $2 a$. On the other hand, a bound $C$ and $\bar{C}$ will have a radius of order $a$. When $R>>a$, there is a mis-match between $R$ and $a$. Because of this mis-match, the probability for the $C$ to interact with the $\bar{C}$ to form a bound color-singlet $C \bar{C}$ after the exchange of a single gluon, as depicted in Fig. $2 a$, is highly suppressed. The estimate of Swanson[11] on the mixing of the quark-interchange component of Fig. $2 a$ (using a superposition of quark-based and meson-based models) gives a mixing probability ranging from zero percent to a maximum of 17 percent. For $R>>a$, this one-gluon exchange contribution as represented by Fig $2 a$ is not the dominant process. Within a quark-based model, far more likely after the exchange of a single gluon is the exchange of an additional gluon from one meson to the other meson to neutralize the octet colors, as depicted in Fig. $2 b$. For our case of $R>>a$, it is reasonable to consider only Fig. $2 b$ as the dominant contribution in the present exploratory work. The contribution of Fig. $2 a$ can be included in future refinements.

The time scale for a meson to be in a color-octet state after emitting a single gluon, which can be called the coloroctet persistence time, is of order $\hbar /\left(\alpha_{s} / a\right)$ [53]. The time scale for molecular motion is of order $\hbar / \epsilon$. Therefore, the color-octet persistence time is much shorter than the time for molecular motion. As a consequence, mesons undergoing molecular motion are predominantly in their color-singlet state. We can invoke arguments analogous to those used previously by Applequist et al. [52] and Peskin and Bhanot et al. [53-55] to treat the many gluons emitted from a meson to the other meson during the short color-octet persistence period as grouping together into clusters, and each multi-gluon cluster summing to a total color-singlet.

Peskin evaluated the set of 10 two-gluon emission diagrams which occur in the short color-octet persistence period [53]. He showed that in order to obtain gauge invariant results, the gluons originating from a meson are emitted not only from the quark and the antiquark of the meson, but also from the gluons exchanged between the quark and the antiquark within the meson. After the QCD two-gluon emission diagrams from a meson have been summed, the coupling of gluons to a static meson source is similar to the familiar coupling of QED photons through simple QED electric dipole interactions. The interaction between mesons can be approximated by a dipole-dipole interaction as in the van der Waals interaction between electric dipoles [53-55]. The only new feature is that the quark and antiquark of the meson is bound into a dipole pair with effective charges for the quark and the antiquark. These similarities will allow us to introduce the effective charges in a QED-type interaction to describe the interaction between constituents in different mesons.

We can examine the results of Peskin and Bhanot to motivate our introduction of the effective charges. In QCD, after the 10 two-gluon emission diagrams have been added together, Peskin [53] and Bhanot and his collaborators $[54,55]$ obtained the gauge-invariant result that the square of the color-electric field strength at a distance $r$ changes from the $r^{-7}$ behavior at very large distances to a $r^{-6}$ behavior at intermediate distance, in the same way as the Casimir-Polder effect in atomic physics [61]. At a distance $r$ with $a / \alpha_{s}>r>a$, in the vicinity of a color-singlet quark-antiquark meson represented by a Wilson loop, the square of the color-electric field strength is [53-55]

$$
\left\langle\boldsymbol{E}^{2}(\boldsymbol{r})\right\rangle=\frac{C_{2} \alpha_{s} a^{2}}{4 \pi r^{6}}\left(1+3 \cos ^{2} \theta\right),
$$

where $C_{2}=\left(N^{2}-1\right) / 2 N$ is the Casimir eigenvalue for the fundamental representation of the $\mathrm{SU}(\mathrm{N})_{\text {color }}$ group, $\alpha_{s}=g^{2} / 4 \pi, g$ is the strong interaction coupling constant, and $\theta$ is the angle between $\boldsymbol{a}$ and $\boldsymbol{r}$. On the other hand, 
the square of the electric field strength in QED at a distance $r$, with $a / \alpha>r>a$, in the vicinity of an electric charge dipole represented by a Wilson loop, is [55] (see also [62])

$$
\left\langle\boldsymbol{E}_{\mathrm{QED}}^{2}(\boldsymbol{r})\right\rangle=\frac{\alpha a^{2}}{4 \pi r^{6}}\left(1+3 \cos ^{2} \theta\right),
$$

where $\alpha=e^{2} / 4 \pi$ is the fine-structure constant. Therefore, if the quark has an effective charge $c_{q}=\sqrt{C_{2}}$ (in unit of $g$ ) and the antiquark an equal and opposite effective charge $c_{\bar{q}}=-\sqrt{C_{2}}$ (in unit of $g$ ), and each effective charge generates a color electric field strength as as in QED, then the square of the total color electric field strength $\boldsymbol{E}^{2}$ in the vicinity of a meson will be the same as given in Eq. (23) obtained by summing up two-gluon emission diagrams in QCD. Because of the similarities between Eqs. (23) and (24), effective charges in a QED-type interaction is a simple way to represent multi-quark QCD interactions in the vicinity of a meson. At short distances, the effective charges in a QED-type interaction also leads to the proper QCD color-Coulomb interaction between a quark and an antiquark in a color-singlet state

$$
V_{c}(r)=-\frac{C_{2} \alpha_{s}}{r}
$$

The interaction between a quark and an antiquark contains not only of the color-Coulomb component but also the linear confining potential. We need to modify the color-Coulomb interaction to include both the color-Coulomb potential and the linear confining potential. Recent lattice gauge calculations show that the nonperturbative interquark potential between a quark and an antiquark in different representations is proportional to the eigenvalue of the quadratic Casimir operator $C_{2}$ [63]. Thus, there is the Casimir scaling in the interaction of a quark and an antiquark and the concept of the effective charge is also applicable when the potential is generalized to include both the color-Coulomb and the confining linear components.

Color interaction given by Eq. (23) is the well-known van der Waals interactions arising from repeated applications of the one-gluon exchange interaction for extended and separated mesons [50-55,60]. There is however no experimental evidence for the inverse-power color van der Waals interaction between separated mesons. This experimental contradiction led Lipkin and Greenberg [50] to note that the quark degree of freedom with only one gluon-exchange interaction for a multi-quark system is incomplete. The gluon in non-Abelian QCD with its color charges and nonlinear self-interaction is very different from the neutral photon. The gluonic degree of freedom is needed in multi-quark interactions [50]. One can represent the gluonic degree of freedom in terms of a string, and the important effect of the dynamical string is the breaking of the string at large distances, resulting in the screening of the long-range interaction. Accordingly, we should modify the phenomenological "color-Coulomb plus linear" interaction further by introducing a screening mass $\mu$, because we intend to use the effective interaction also for distances larger than $1 \mathrm{fm}$ for our molecular state.

When Eq. (25) is written as $V_{c}(r)=-C_{2} v_{c}(r)$ by separating out the color factor $\left(-C_{2}\right)$, the basic color-Coulomb interaction is $v_{c}(r)=\alpha_{s} / r$ and the corresponding basic linear confining interaction is $v_{\text {lin }}(r)=-3 b r / 4$. They can be represented in the momentum space by $\tilde{v}_{c}(\boldsymbol{k})=4 \pi \alpha_{s} / \boldsymbol{k}^{2}$ and $\tilde{v}_{\text {lin }}(\boldsymbol{k})=6 \pi b / \boldsymbol{k}^{4}$. The effect of screening can be introduced by replacing $\boldsymbol{k}^{2}$ with $\boldsymbol{k}^{2}+\mu^{2}$, leading to an interaction in momentum space given by [64]

$$
\widetilde{v}(\mathbf{k})=\left[\frac{4 \pi \alpha_{s}}{\mathbf{k}^{2}+\mu^{2}}+\frac{6 \pi b}{\left(\mathbf{k}^{2}+\mu^{2}\right)^{2}}\right] .
$$

From the interaction in momentum space, we can obtain the interaction in the the configuration space. The screened color-Coulomb interaction becomes the Yukawa interaction, and the screened linear potential becomes the exponential interaction. One also needs to include the hyperfine spin-spin interaction. As the spin-spin interaction is short-ranged, the effect of screening on the spin-spin interaction can be neglected.

The effective interaction between quark and antiquark particles $j$ and $k$ in the four-body system is then given by

$$
V_{j k}\left(\boldsymbol{r}_{j k}\right)=c_{j} c_{k} v\left(\boldsymbol{r}_{j k}\right)
$$

where

$$
c_{j}=\left\{\begin{aligned}
\sqrt{C_{2}} & \text { if } j \text { is a quark, } \\
-\sqrt{C_{2}} & \text { if } j \text { is an antiquark }
\end{aligned}\right.
$$

and

$$
v\left(\boldsymbol{r}_{j k}\right)=\frac{\alpha_{s} e^{-\mu r_{j k}}}{r_{j k}}+\frac{3 b}{4 \mu} e^{-\mu r_{j k}}-\frac{8 \pi \alpha_{s}}{3 m_{j} m_{k}} \boldsymbol{s}_{j} \cdot \boldsymbol{s}_{k}\left(\frac{\sigma^{3}}{\pi^{3 / 2}}\right) e^{-\sigma^{2} r_{j k}^{2}}
$$


The central potential reduces to the usual Cornell-type potential in the limit when $\mu \rightarrow 0$. With this effective interaction, a quark and an antiquark in the four-body molecular state $(Q \bar{q})-(q \bar{Q})$ system are subject to the same phenomenological interaction and can lead to the proper meson bound states.

The residue interaction between $A(12)$ and $B(34)$ is then

$$
V_{I}=\sum_{j=1}^{2} \sum_{k=3}^{4} c_{j} c_{k} v\left(\boldsymbol{r}_{j k}\right)
$$

By making use of earlier studies of potential parameters and a running coupling constant [42], we use an effective interaction with the following set of parameters for our present work

$$
\begin{aligned}
\alpha_{s}\left(Q^{2}\right) & =\frac{12 \pi}{\left(33-2 n_{f}\right) \ln \left(A+Q^{2} / B^{2}\right)}, \quad A=10, \quad B=0.31 \mathrm{GeV}, \\
b & =0.335 \mathrm{GeV}^{2}, \quad \mu=0.28 \mathrm{GeV}, \quad \sigma=0.897 \mathrm{GeV}, \\
m_{u} & =m_{d}=0.334 \mathrm{GeV}, \quad m_{c}=1.87 \mathrm{GeV}, \quad m_{b}=5.18 \mathrm{GeV} .
\end{aligned}
$$

The string tension coefficient $b=0.335 \mathrm{GeV}^{2}$ and the screening mass $\mu=0.28 \mathrm{GeV}$ were previously found to give reasonable descriptions of the charmonium masses [65]. The value of the screening mass gives a screening length of $1 / \mu \sim 0.7 \mathrm{fm}$ which is consistent with the minimum string-breaking distance $L_{\min }$, (Eq. (5.11) of [66]), based on the Schwinger mechanism [67] of pair production,

$$
L_{\text {min }}=2 m_{u, d} / \kappa=0.67 \mathrm{fm},
$$

where we have used a string tension coefficient $\kappa$ of $1 \mathrm{GeV} / \mathrm{fm}$. The large value of $b$ in Eq. (31) arises because the effective string tension coefficient $b e^{-\mu r_{j k}}$ is not constant and it varies with $r_{j k}$. In Eq. (31), we identify $Q$ as the sum of the rest masses of the interacting quarks. The parameterization of the phenomenological strong coupling constant $\alpha_{s}$ and the spin-spin interaction parameter $\sigma$ come from earlier calculations of meson masses in [42]. The set of parameters in Eq. (31) leads to a good description of the masses of $D, D^{*}, J / \psi, B, B^{*}$, and $\Upsilon$ mesons.

\section{EVALUATION OF THE MESON-MESON POTENTIAL $V(r)$}

We represent the wave function for the coordinate of the quark $Q$ relative to the antiquark $\bar{q}$ in the meson $A(12)$ by a Gaussian wave function of the form

$$
\phi_{l m}^{A}\left(\boldsymbol{r}_{12}\right)=\sqrt{\frac{4 \pi}{(2 l+1) ! !}}\left(\frac{\beta_{A}^{2}}{\pi}\right)^{3 / 4}\left(2 \beta_{A}^{2}\right)^{l / 2} i^{l} r_{12}^{l} e^{-\beta_{A}^{2} r_{12}^{2} / 2} Y_{l m}\left(\theta_{12}, \phi_{12}\right) .
$$

The wave function is normalized according to

$$
\int d \boldsymbol{r}\left|\phi_{l m}^{A}\left(\boldsymbol{r}_{12}\right)\right|^{2}=1
$$

A similar wave function $\phi_{l^{\prime} m^{\prime}}^{B}\left(\boldsymbol{r}_{34}\right)$ can be written for the relative motion between the quark $q$ and the antiquark $\bar{Q}$ in the meson $B(34)$.

With the knowledge of the Gaussian wave functions and the effective interquark interaction, the meson-meson potential $V(r)$ can be evaluated analytically. The meson-meson potential depends on $\left\langle A_{\lambda} B_{\lambda^{\prime}}\left|V_{I}\right| A_{0} B_{0}\right\rangle$ which can be further decomposed as a sum of $\left\langle A_{\lambda} B_{\lambda^{\prime}}\left|v\left(\boldsymbol{r}_{j k}\right)\right| A_{0} B_{0}\right\rangle$,

$$
\left\langle A_{\lambda} B_{\lambda^{\prime}}\left|V_{I}\right| A_{0} B_{0}\right\rangle=\sum_{j=1}^{2} \sum_{k=3}^{4} c_{j} c_{k}\left\langle A_{\lambda} B_{\lambda^{\prime}}\left|v\left(\boldsymbol{r}_{j k}\right)\right| A_{0} B_{0}\right\rangle .
$$

The matrix elements $\left\langle A_{\lambda} B_{\lambda^{\prime}}\left|v\left(\boldsymbol{r}_{j k}\right)\right| A_{0} B_{0}\right\rangle$ can be evaluated in the configuration space where there are three independent coordinates because the coordinates of the four particles are connected by the center-of-mass coordinate $\boldsymbol{R}_{1234}$. We can fix the center-of-mass coordinate $\boldsymbol{R}_{1234}$ to be at the origin,

$$
\boldsymbol{R}_{1234}=\left(m_{12} \boldsymbol{R}_{12}+m_{34} \boldsymbol{R}_{34}\right) /\left(m_{12}+m_{34}\right)=\mathbf{0},
$$


and choose the independent coordinates to be $\boldsymbol{r}, \boldsymbol{r}_{12}$, and $\boldsymbol{r}_{34}$. Using Eqs. (2), (5), (6), (15), and (36), we can represent all other coordinates in terms of these three coordinates:

$$
\boldsymbol{r}_{j k}=\boldsymbol{r}+f_{A}(j k) \boldsymbol{r}_{12}+f_{B}(j k) \boldsymbol{r}_{34}
$$

where

$$
\begin{aligned}
& f_{A}(14)=f_{2}, \quad f_{B}(14)=f_{3}, \\
& f_{A}(13)=f_{2}, \quad f_{B}(13)=-f_{4}, \\
& f_{A}(23)=-f_{1}, \quad f_{B}(23)=-f_{4}, \\
& f_{A}(24)=-f_{1}, \quad f_{B}(24)=f_{3} .
\end{aligned}
$$

Using the above relations between $\boldsymbol{r}_{j k}$ and $\left\{\boldsymbol{r}, \boldsymbol{r}_{12}, \boldsymbol{r}_{34}\right\}$, the matrix element $\left\langle A_{\lambda} B_{\lambda^{\prime}}\left|v\left(\boldsymbol{r}_{j k}\right)\right| A_{0} B_{0}\right\rangle$ can be written as

$$
\left\langle A_{\lambda} B_{\lambda^{\prime}}\left|v\left(\boldsymbol{r}_{j k}\right)\right| A_{0} B_{0}\right\rangle=\int d \boldsymbol{r}_{12} \int d \boldsymbol{r}_{34} \rho_{\lambda 0}^{A}\left(\boldsymbol{r}_{12}\right) \rho_{\lambda^{\prime} 0}^{B}\left(\boldsymbol{r}_{34}\right) v\left(\boldsymbol{r}+f_{A}(j k) \boldsymbol{r}_{12}+f_{B}(j k) \boldsymbol{r}_{34}\right),
$$

where $\rho_{\lambda 0}^{A}$ and $\rho_{\lambda^{\prime} 0}^{B}$ are

$$
\begin{gathered}
\rho_{\lambda 0}^{A}\left(\boldsymbol{r}_{12}\right)=\phi_{\lambda}^{*}\left(\boldsymbol{r}_{12}\right) \phi_{0}\left(\boldsymbol{r}_{12}\right), \\
\rho_{\lambda^{\prime} 0}^{A}\left(\boldsymbol{r}_{34}\right)=\phi_{\lambda^{\prime}}^{*}\left(\boldsymbol{r}_{34}\right) \phi_{0}\left(\boldsymbol{r}_{34}\right) .
\end{gathered}
$$

We note that $\rho_{00}^{A}\left(\boldsymbol{r}_{12}\right)$ is the density distribution of meson $A_{0}(12)$ and $\rho_{\lambda 0}^{A}\left(\boldsymbol{r}_{12}\right)$ is the transition density for making the transition from the ground state $A_{0}(12)$ to the excited states $A_{\lambda}(12)$. Similarly, $\rho_{00}^{B}\left(\boldsymbol{r}_{34}\right)$ and $\rho_{\lambda^{\prime} 0}^{B}\left(\boldsymbol{r}_{12}\right)$ are respectively the density distribution and transition density of meson $B(12)$. By the method of Fourier transform [58, 68], the evaluation of the matrix element (39) can be greatly simplified. The result is

$$
\left\langle A_{\lambda} B_{\lambda^{\prime}}\left|v\left(\boldsymbol{r}_{j k}\right)\right| A_{0} B_{0}\right\rangle=\int \frac{d \boldsymbol{p}}{(2 \pi)^{3}} e^{i \boldsymbol{p} \cdot \boldsymbol{r}} \tilde{\rho}_{\lambda 0}^{A}\left[f_{A}(j k) \boldsymbol{p}\right] \quad \tilde{\rho}_{\lambda^{\prime} 0}^{B}\left[f_{B}(j k) \boldsymbol{p}\right] \tilde{v}(\boldsymbol{p})
$$

where

$$
\begin{aligned}
& \tilde{\rho}_{\lambda 0}^{A, B}(\boldsymbol{p})=\int d \boldsymbol{y} e^{i \boldsymbol{p} \cdot \boldsymbol{y}} \rho_{\lambda 0}^{A, B}(\boldsymbol{y}), \\
& \tilde{v}(\boldsymbol{p})=\int d \boldsymbol{r}_{j k} e^{-i \boldsymbol{p} \cdot \boldsymbol{r}_{j k}} v\left(\boldsymbol{r}_{j k}\right) .
\end{aligned}
$$

We first evaluate the matrix element $\left\langle A_{0} B_{0}\left|v\left(\boldsymbol{r}_{j k}\right)\right| A_{0} B_{0}\right\rangle$ which is also needed in subsequent calculations. From the wave functions we can determine

$$
\tilde{\rho}_{00}^{A, B}(\boldsymbol{p})=e^{-\boldsymbol{p}^{2} / 4 \beta_{A, B}^{2}} .
$$

Therefore, the matrix element $\left\langle A_{0} B_{0}\left|v\left(\boldsymbol{r}_{j k}\right)\right| A_{0} B_{0}\right\rangle$ is related to $\tilde{v}(\boldsymbol{p})$ by

$$
\left\langle A_{0} B_{0}\left|v\left(\boldsymbol{r}_{j k}\right)\right| A_{0} B_{0}\right\rangle=\int \frac{p^{2} d p d \mu d \phi}{(2 \pi)^{3}} e^{i \boldsymbol{p} \cdot \boldsymbol{r}} e^{-a_{j k}^{2} p^{2}} \tilde{v}(p) \equiv v_{00}\left(j k ; a_{j k}^{2}, r\right),
$$

where $a_{j k}^{2}$ is

$$
a_{j k}^{2}=\frac{\left[f_{A}(j k)\right]^{2}}{4 \beta_{A}^{2}}+\frac{\left[f_{B}(j k)\right]^{2}}{4 \beta_{B}^{2}}
$$

and we have written $\left\langle A_{0} B_{0}\left|v\left(\boldsymbol{r}_{j k}\right)\right| A_{0} B_{0}\right\rangle$ as $v_{00}\left(j k ; a_{j k}^{2}, r\right)$ to exhibit fully its dependence on $a_{j k}^{2}$ and $r$.

The matrix element $\left\langle A_{0} B_{0}\left|v\left(\boldsymbol{r}_{j k}\right)\right| A_{0} B_{0}\right\rangle$ can be expressed as the sum of contributions from the Yukawa interaction and the exponential interaction in Eq. (29),

$$
\left\langle A_{0} B_{0}\left|v\left(\boldsymbol{r}_{j k}\right)\right| A_{0} B_{0}\right\rangle=\left\langle A_{0} B_{0}\left|v^{\mathrm{Yuk}}\left(\boldsymbol{r}_{j k}\right)\right| A_{0} B_{0}\right\rangle+\left\langle A_{0} B_{0}\left|v^{\exp }\left(\boldsymbol{r}_{j k}\right)\right| A_{0} B_{0}\right\rangle .
$$


The matrix element for the Yukawa interaction is found to be

$$
\begin{gathered}
\left\langle A_{0} B_{0}\left|v^{\mathrm{Yuk}}\left(\boldsymbol{r}_{j k}\right)\right| A_{0} B_{0}\right\rangle=\alpha_{s} e^{a_{j k}^{2} \mu^{2}}\left(u_{1} v_{1}-u_{2} v_{2}\right), \\
u_{1}=\left(1+\operatorname{erf}\left[\left(r-2 a_{j k}^{2} \mu\right) /\left(2 a_{j k}\right)\right]\right) / 2, \\
u_{2}=\left(1-\operatorname{erf}\left[\left(r+2 a_{j k}^{2} \mu\right) /\left(2 a_{j k}\right)\right]\right) / 2, \\
v_{1}=\frac{e^{-\mu r}}{r} \\
v_{2}=\frac{e^{\mu r}}{r} .
\end{gathered}
$$

The matrix element for the exponential interaction is

$$
\left\langle A_{0} B_{0}\left|v^{\exp }\left(\boldsymbol{r}_{j k}\right)\right| A_{0} B_{0}\right\rangle=\frac{3 b}{4 \mu} e^{a_{j k}^{2} \mu^{2}}\left(u_{1} w_{1}+u_{2} w_{2}\right),
$$

where

$$
w_{1}=\left(r-2 a_{j k}^{2} \mu\right) \frac{e^{-\mu r}}{r}
$$

and

$$
w_{2}=\left(r+2 a_{j k}^{2} \mu\right) \frac{e^{\mu r}}{r} .
$$

The direct potential vanishes when all the quark masses are equal, and it becomes more attractive as the mass of the heavy quark increases.

We fix the vector $\boldsymbol{r}$ to lie along the $z$-axis, and quantize the azimuthal component of the angular momentum $m$ to be projections along the $z$-axis. For the polarization potential $V_{\text {pol }}(r)$, we consider intermediate excitations to the next excited states of $A(12)$ and $B(12)$ characterized by $\left\{\lambda\right.$ or $\left.\lambda^{\prime}=l m=1 m\right\}$. It can be shown that the matrix element $\left\langle A_{\lambda} B_{\lambda^{\prime}}\left|v\left(\boldsymbol{r}_{j k}\right)\right| A_{0} B_{0}\right\rangle$ is zero for $\left\{\lambda=0 ; \lambda^{\prime}=1 m\right\}$ and $\left\{\lambda=1 m ; \lambda^{\prime}=0\right\}$. It gives a non-vanishing matrix element for $\left\{\lambda=1 m ; \lambda^{\prime}=1-m\right\}$ with $\{m=-1,0,1\}$ when both mesons are excited.

To evaluate the matrix element $\left\langle A_{1 m} B_{1-m}\left|v\left(\boldsymbol{r}_{j k}\right)\right| A_{0} B_{0}\right\rangle$, we write out the wave functions explicitly and obtain

$$
\left\langle A_{10} B_{10}\left|v\left(\boldsymbol{r}_{j k}\right)\right| A_{0} B_{0}\right\rangle=\frac{f_{A}(j k) f_{B}(j k)}{2 \beta_{A} \beta_{B}} \int \frac{p^{2} d p d \mu d \phi}{(2 \pi)^{2}} e^{i \boldsymbol{p} \cdot \boldsymbol{r}} e^{-a_{j k}^{2} p^{2}} p^{2} \mu^{2} \tilde{v}(p) .
$$

A comparison of the above equation with Eq. (46) yields

$$
\left\langle A_{10} B_{10}\left|v\left(\boldsymbol{r}_{j k}\right)\right| A_{0} B_{0}\right\rangle=-\frac{f_{A}(j k) f_{B}(j k)}{2 \beta_{A} \beta_{B}} \frac{\partial^{2}}{\partial r^{2}} v_{00}\left(j k ; a_{j k}^{2}, r\right) .
$$

To evaluate the matrix element $\left\langle A_{11} B_{1-1}\left|v\left(\boldsymbol{r}_{j k}\right)\right| A_{0} B_{0}\right\rangle$, we write out the wave functions explicitly and obtain

$$
\left\langle A_{11} B_{1-1}\left|v\left(\boldsymbol{r}_{j k}\right)\right| A_{0} B_{0}\right\rangle=\frac{f_{A}(j k) f_{B}(j k)}{2 \beta_{A} \beta_{B}} \int \frac{p^{2} d p d \mu d \phi}{(2 \pi)^{3}} e^{i \boldsymbol{p} \cdot \boldsymbol{r}} e^{-a_{j k}^{2} p^{2}} \frac{p^{2}\left(1-\mu^{2}\right)}{2} \tilde{v}(p) .
$$

A comparison of the above equation with Eq. (46) gives

$$
\left\langle A_{11} B_{1-1}\left|v\left(\boldsymbol{r}_{j k}\right)\right| A_{0} B_{0}\right\rangle=\frac{f_{A}(j k) f_{B}(j k)}{2 \beta_{A} \beta_{B}} \frac{1}{2}\left(-\frac{\partial}{\partial a_{j k}^{2}}+\frac{\partial^{2}}{\partial r^{2}}\right) v_{00}\left(j k ; a_{j k}^{2}, r\right) .
$$

With the analytical results of Eqs. (48), (49), and (54) for $\left\langle A_{0} B_{0}\left|v\left(\boldsymbol{r}_{j k}\right)\right| A_{0} B_{0}\right\rangle$ (or $v_{00}\left(j k ; a_{j k}^{2}, r\right)$ ), the differentiation of $v_{00}\left(j k ; a_{j k}^{2}, r\right)$ with respect to $a_{j k}^{2}$ and $r$ can be readily carried out analytically to give the matrix elements $\left\langle A_{1 m} B_{1-m}\left|v\left(\boldsymbol{r}_{j k}\right)\right| A_{0} B_{0}\right\rangle$ for $\{m=-1,0,1\}$. The energy denominator in Eq. (22), $\left\{\epsilon\left(A_{1 m}\right)+\epsilon\left(B_{1-m}\right)-\epsilon\left(A_{0}\right)-\epsilon\left(B_{0}\right)\right\}$, can be obtained from experimental masses of $D(1 P), \bar{D}^{*}(1 P), D(1 S)$, and $\bar{D}^{*}(1 S)$. The masses of the $1 \mathrm{P}$ states of the open bottom mesons are not known experimentally. We infer the mass differences from theoretical masses of open bottom mesons calculated in [42] where we get $M(B(1 P))-M\left(B_{0}\right)=0.5348 \mathrm{GeV}$, and $M\left(B^{*}(1 P)\right)-M\left(B_{0}^{*}\right)=0.5168$ $\mathrm{GeV}$. These quantities allows us to calculate the polarization potential using Eq. (22). 


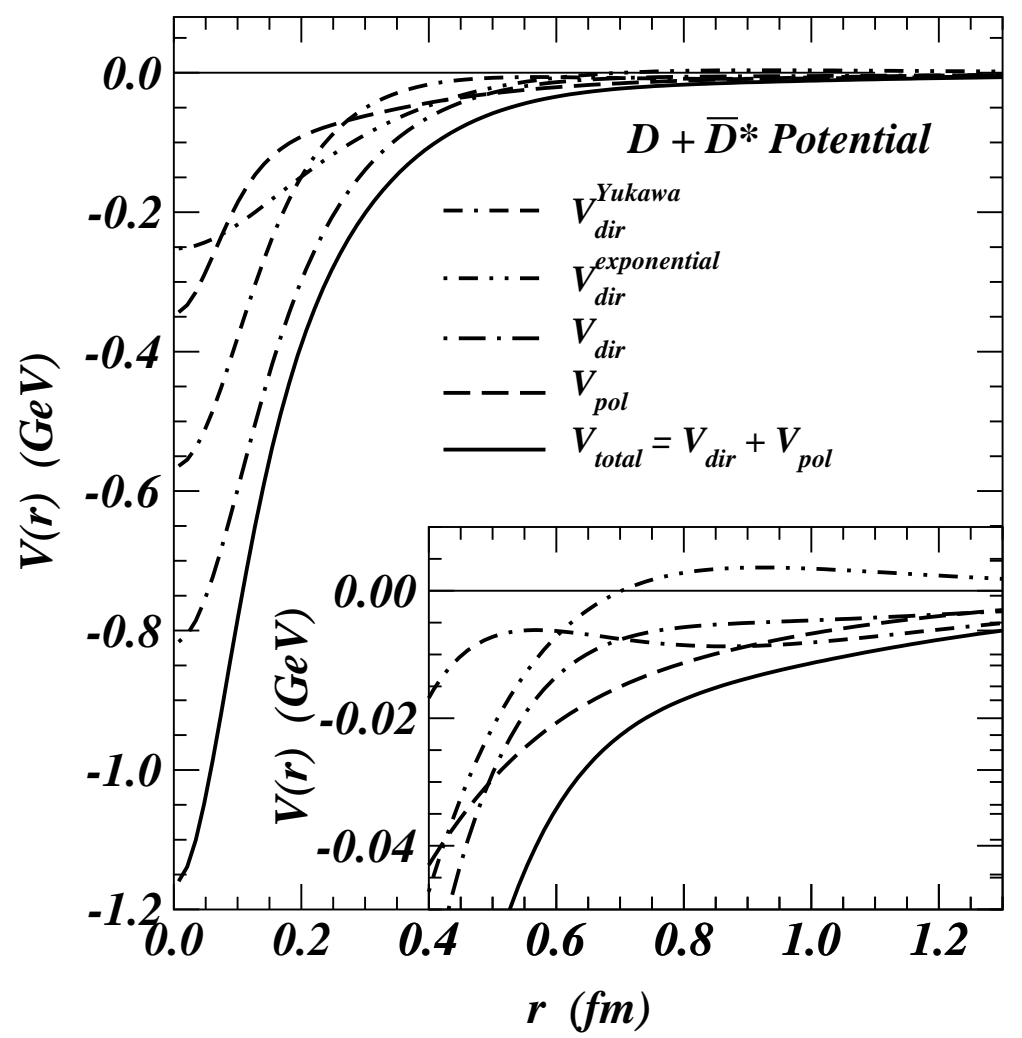

FIG. 3: The total potential $V(r)$ and its various components for the $D \bar{D}^{*}$ system. The direct potential $V_{\text {dir }}$ is the sum of $V_{\text {dir }}^{\text {Yuka }}$ and $V_{\text {dir }}^{\text {exponential }}$, and $V(r)$ is the sum of the direct potential and the polarization potential. The insert with an expanded ordinate scale gives a clearer picture of the total potential and its various components at large distances.

\section{HEAVY QUARK MOLECULAR STATES}

The meson wave function in Eq. (33) is characterized by a momentum width parameter $\beta$ that is related to the root-mean-square quark-antiquark separation $r_{\mathrm{rms}}$ of the meson by

$$
\beta=\sqrt{\frac{3}{2}} \frac{1}{r_{\mathrm{rms}}} .
$$

Previously, root-mean-square quark-antiquark separation of mesons have been obtained from calculations of meson wave functions and meson masses (Table IV of [42]). From this table, we use the root-mean-square quark-antiquark separations of $r_{\mathrm{rms}}(D)=0.585 \mathrm{fm}, r_{\mathrm{rms}}\left(D^{*}\right)=0.626 \mathrm{fm}, r_{\mathrm{rms}}(B)=0.574 \mathrm{fm}$, and $r_{\mathrm{rms}}\left(D^{*}\right)=0.583 \mathrm{fm}$ to calculate the $\beta$ values for various meson wave functions. In the present numerical work, we shall include only the screened color-Coulomb interaction (Yukawa interaction) and the screened linear interaction (exponential interaction) so as to study the gross features of the meson-meson potential and molecular states.

Using the effective interaction outlined above, we obtained meson-meson potentials for various combinations of $D, D^{*}, B, B^{*}$ with $\bar{D}, \bar{D}^{*}, \bar{B}, \bar{B}^{*}$. The total potential $V(r)$ and its various components for $D \bar{D}^{*}$ are shown in Fig. 3 . The insert in Fig. 3 gives a more expanded description of various components at large distances. The total direct potential is the sum of the contributions from the Yukawa and the exponential interaction, and is attractive. The magnitude of the polarization potential is smaller than the magnitude of the direct potential at short distances but the role is reversed at intermediate separations. The two different contributions are about equal at large separations beyond $1.2 \mathrm{fm}$. As a result, the total potential, which is the sum of the direct potential and the polarization potential, has an extended attractive region extending to $1 \mathrm{fm}$ and beyond.

With such a potential and the experimental masses of $D$ and $\bar{D}^{*}$, we solve for the lowest eigenstate of the mesonmeson system. We find a bound state at a binding energy of $7.51 \mathrm{MeV}$. We plot the wave function for this molecular state in Fig. 4. The wave function $\psi(r)$ is quite extended and has a significant amplitude beyond 1 fm. This state is weakly bound, and has a large root-mean-square $D-\bar{D}^{*}$ separation of $1.37 \mathrm{fm}$. Its weak binding and its extended spatial separation between $D$ and $\bar{D}^{*}$ provide a good characterization of its molecular structure. 


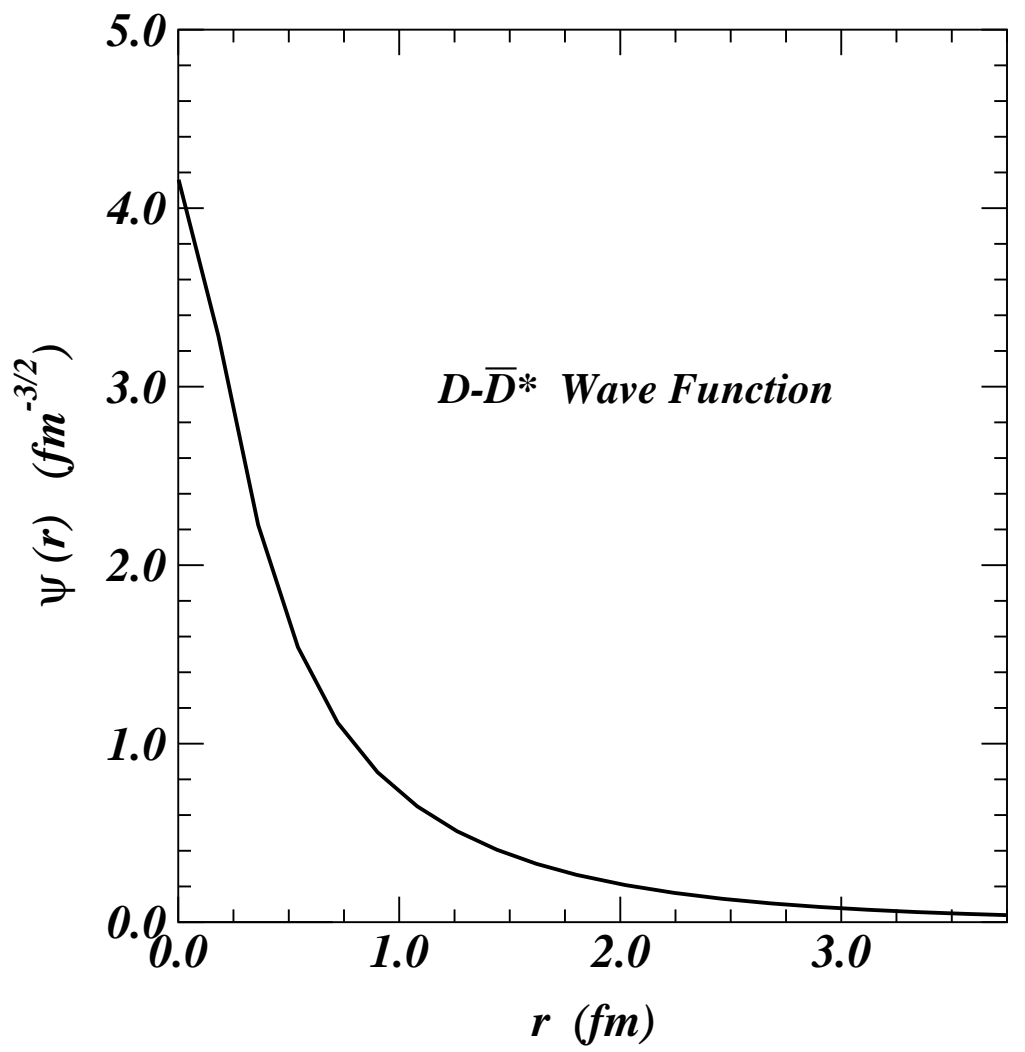

FIG. 4: The wave function $\psi(r)$ of the $D \bar{D}^{*}$ system as a function of the separation between $D$ and $\bar{D}^{*}$.

For a flavor-independent effective interaction, the theoretical meson-meson potentials and the binding energies are expected to change very insignificantly for different flavors of the light quarks. On the other hand, the mass of the system is related to the binding energy $B$ by

$$
M=M\left(A_{\nu}\right)+M\left(B_{\nu^{\prime}}\right)+\epsilon=M\left(A_{\nu}\right)+M\left(B_{\nu^{\prime}}\right)-B,
$$

and the masses of $A_{\nu}$ and $B_{\nu^{\prime}}$ depend on light quark flavors. Therefore, the calculated mass of the $(Q \bar{q})-(q \bar{Q})$ molecular system depends on light quark flavors. We list the molecular states for different combinations of the $(c \bar{q})$ and $(q \bar{c})$ mesons in Table I. The properties of the states in the $D \bar{D}, D \bar{D}^{*}$, and $D^{*} \bar{D}^{*}$ systems are quite similar.

Table I. The calculated masses, binding energies, and the root-mean-square $(c \bar{q})-(q \bar{c})$ separation $r_{\text {rms }}$ of molecular states formed by $(c \bar{q})$ and $(q \bar{c})$ mesons.

\begin{tabular}{|c|c|c|c|c|}
\hline$(c \bar{q})-(q \bar{c})$ & State & Binding Energy $(\mathrm{MeV})$ & Mass $(\mathrm{MeV})$ & $r_{\text {rms }}(\mathrm{fm})$ \\
\hline$D^{0} \bar{D}^{0}$ & $1 \mathrm{~S}$ & 3.10 & 3725.90 & 1.73 \\
$D^{0} D^{-}, D^{+} \bar{D}^{0}$ & $1 \mathrm{~S}$ & 3.10 & 3730.70 & 1.73 \\
$D^{+} D^{-}$ & $1 \mathrm{~S}$ & 3.10 & 3735.50 & 1.73 \\
\hline$D^{0} \bar{D}^{* 0}$ & $1 \mathrm{~S}$ & 7.53 & 3863.67 & 1.37 \\
$D^{0} D^{*-}$ & $1 \mathrm{~S}$ & 7.53 & 3866.97 & 1.37 \\
$D^{+} \bar{D}^{* 0}$ & $1 \mathrm{~S}$ & 7.53 & 3868.47 & 1.37 \\
$D^{+} D^{*-}$ & $1 \mathrm{~S}$ & 7.53 & 3871.77 & 1.37 \\
\hline$D^{* 0} \bar{D}^{* 0}$ & $1 \mathrm{~S}$ & 15.31 & 3998.09 & 1.06 \\
$D^{* 0} D^{*-}, D^{*+} \bar{D}^{* 0}$ & $1 \mathrm{~S}$ & 15.31 & 4001.39 & 1.06 \\
$D^{*+} D^{*-}$ & $1 \mathrm{~S}$ & 15.31 & 4004.69 & 1.06 \\
\hline
\end{tabular}

To produce the unknown state $X(3872)$ in the process $B^{ \pm} \rightarrow K^{ \pm} X(3872)$ observed by the Belle Collaboration, the state $X(3872)$ most likely originates from the production of a $c \bar{c}$ pair with $I=0$ during the weak decay of the bottom 
quark. On the other hand, the calculated masses of $D^{0} \bar{D}^{* 0}$ and $D^{+} D^{*-}$ molecular systems are respectively 3863.67 $\mathrm{MeV}$ and $3871.77 \mathrm{MeV}$ (see Table I). By the symmetry of charge conjugation, the masses of $D^{* 0} \bar{D}^{0}$ and $D^{-} D^{*+}$ systems are also $3863.67 \mathrm{MeV}$ and $3871.77 \mathrm{MeV}$ respectively. The most suitable candidate for the $3872 \mathrm{MeV}$ state appears to be the molecular state $D^{+} D^{*-}$ and its charge conjugate $D^{-} D^{*+}$. One expects that the molecular states $D^{0} \bar{D}^{* 0}, D^{+} \bar{D}^{*-}$, and $D^{-} D^{*+}$ will be mixed to form components of $I=0$ and $I=1$ states. As they lie in the vicinity of the 3872 state, it is tempting to identify the $I=0$ of this set of multiplets as the 3872 state observed by the Belle Collaboration. It has been conjectured however that the 3872 state may break isospin symmetry maximally [4, 6] and more experimental information are needed to understand better the isospin properties of the 3872 state.

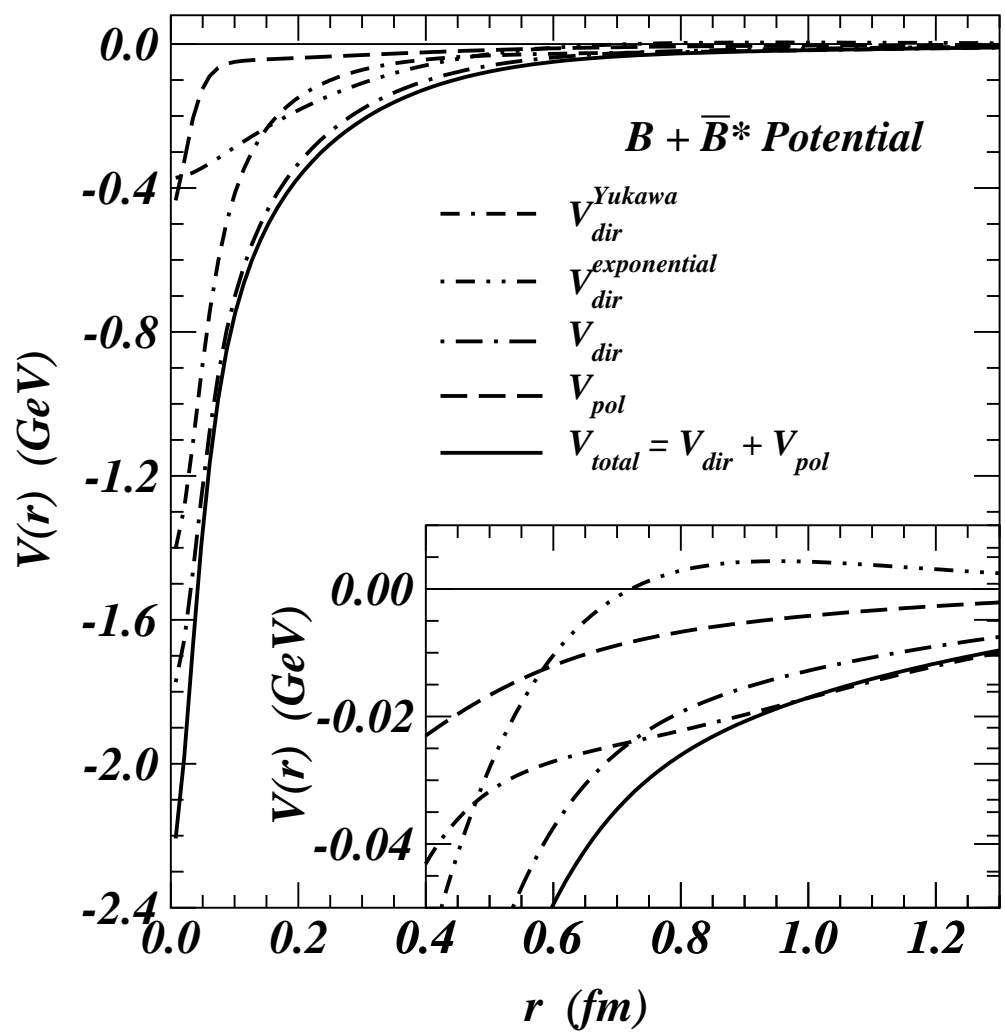

FIG. 5: The total potential $V(r)$ between $B$ and $\bar{B}^{*}$ and its various components. The insert gives the total potential and its various components at large separations in greater detail.

We show in Fig. 5 the potential for the $B \bar{B}^{*}$ system. The potential has features similar to those of the $D \bar{D}^{*}$ system except that the $B \bar{B}^{*}$ potential is deeper than the $D \bar{D}^{*}$ potential. This arises because the quark mass ratio $m_{b} / m_{u, d}$ is greater than $m_{c} / m_{u, d}$. For the $B \bar{B}^{*}$ system, the magnitude of the direct potential is consistently much larger than the magnitude of the polarization potential. The total potential is attractive at large separations. We search for eigenstates for the $B \bar{B}^{*}$ system. The large mass of the bottom quark and the large depth of the $B \bar{B}^{*}$ potential leads to two eigenstates of the $B \bar{B}^{*}$ system. There is a $1 \mathrm{~S}$ state with a binding energy of $151.75 \mathrm{MeV}$ and a root-mean-square $B-\bar{B}^{*}$ separation of $0.314 \mathrm{fm}$. In addition, there is a $2 S$ state with a binding energy of $0.88 \mathrm{MeV}$ and a root-mean-square $B-\bar{B}^{*}$ separation of $2.23 \mathrm{fm}$.

The wave functions for these two states are shown in Fig. 6. The $\psi_{1 S}(r)$ wave function is confined at short distances, but the $\psi_{2 S}(r)$ wave function is spatially quite extended. We list in Table II the calculated binding energies, masses, and the root-mean-square radii of states formed by $(b \bar{q})$ and $(q \bar{b})$. As the masses of the $B^{0}$ and $B^{ \pm}$are nearly the same, we do not need to distinguish $B$ (and similarly $B^{*}$ ) mesons of different light quark flavors.

Table II. The calculated masses, binding energies, and the root-mean-square $(b \bar{q})-(q \bar{b})$ separation $r_{\text {rms }}$ of molecular 
states formed by $(b \bar{q})$ and $(q \bar{b})$ mesons.

\begin{tabular}{|c|c|c|c|c|}
\hline$(b \bar{q})-(q \bar{b})$ & State & Binding Energy $(\mathrm{MeV})$ & Mass $(\mathrm{MeV})$ & $r_{\mathrm{rms}}(\mathrm{fm})$ \\
\hline$B \bar{B}$ & $1 \mathrm{~S}$ & 148.24 & 10410.56 & 0.317 \\
$B \bar{B}^{*}$ & $1 \mathrm{~S}$ & 151.75 & 10452.64 & 0.314 \\
$B^{*} \bar{B}^{*}$ & $1 \mathrm{~S}$ & 155.40 & 10494.60 & 0.311 \\
$B \bar{B}$ & $2 \mathrm{~S}$ & 0.80 & 10558.00 & 2.25 \\
$B \bar{B}^{*}$ & $2 \mathrm{~S}$ & 0.88 & 10603.52 & 2.23 \\
$B^{*} \bar{B}^{*}$ & $2 \mathrm{~S}$ & 0.95 & 10649.05 & 2.21 \\
\hline
\end{tabular}

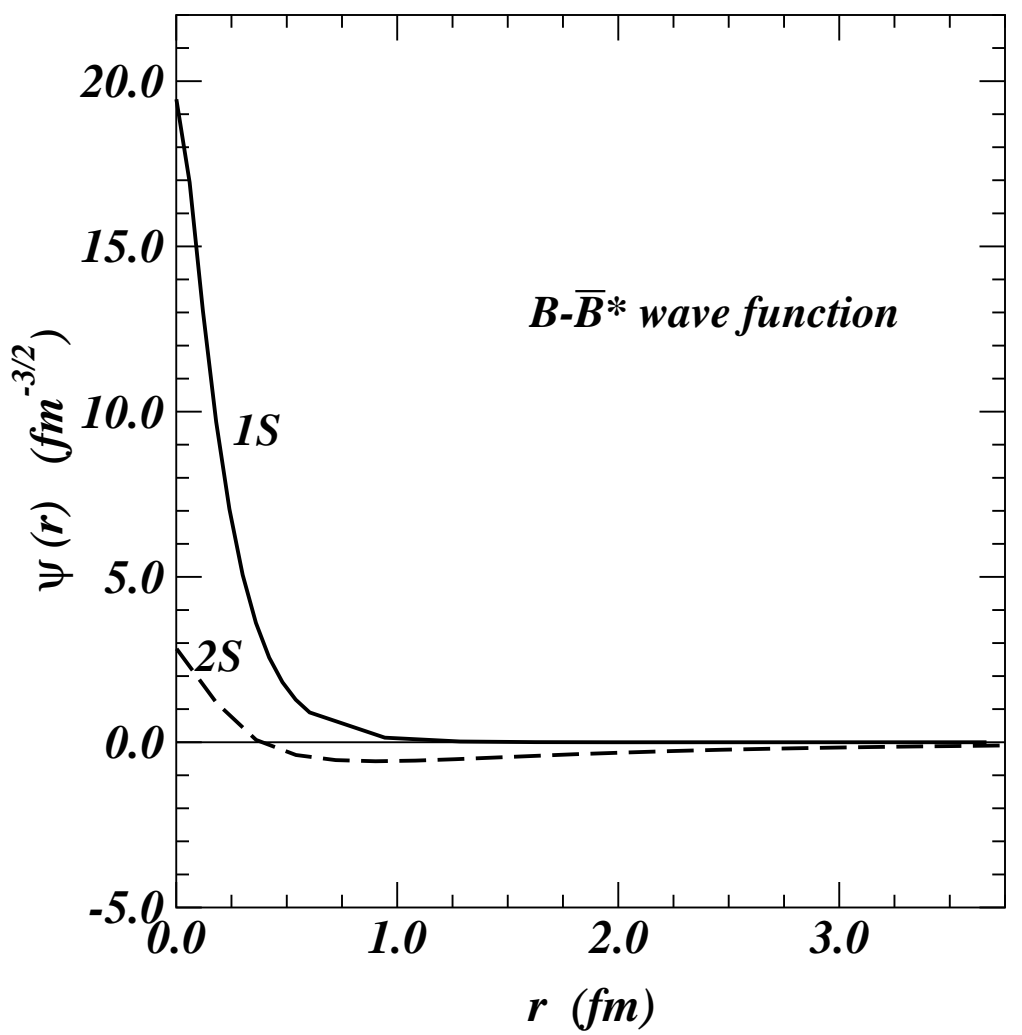

FIG. 6: The wave function $\psi(r)$ of the $B \bar{B}^{*}$ system as a function of the separation between $B$ and $\bar{B}^{*}$.

The potentials and wave functions for $(c \bar{q})-(q \bar{b})$ systems involving both charm quark and bottom quarks are similar to those of $B \bar{B}^{*}$ and $D \bar{D}^{*}$ and have similar $1 S$ eigenstates with a binding energy about $29-43 \mathrm{MeV}$ and a root-meansquare $(c \bar{q})-(q \bar{b})$ separation about $0.6-0.8 \mathrm{fm}$. We list in Table III the eigenstates for various $(c \bar{q})-(q \bar{b})$ systems.

Table III. The calculated masses, binding energies, and the root-mean-square $(c \bar{q})-(q \bar{b})$ separation $r_{\text {rms }}$ of molecular states formed by $(c \bar{q})$ and $(q \bar{b})$ mesons.

\begin{tabular}{|c|c|c|c|c|}
\hline$(c \bar{q})-(q \bar{b})$ & State & Binding Energy $(\mathrm{MeV})$ & Mass $(\mathrm{MeV})$ & $r_{\mathrm{rms}}(\mathrm{fm})$ \\
\hline$D^{0} \bar{B}$ & $1 \mathrm{~S}$ & 27.83 & 7115.01 & 0.756 \\
$D^{+} \bar{B}$ & $1 \mathrm{~S}$ & 27.83 & 7119.81 & 0.756 \\
\hline$D^{0} \bar{B}^{*}$ & $1 \mathrm{~S}$ & 29.36 & 7158.96 & 0.739 \\
$D^{+} \bar{B}^{*}$ & $1 \mathrm{~S}$ & 29.36 & 7163.76 & 0.739 \\
\hline$D^{* 0} \bar{B}$ & $1 \mathrm{~S}$ & 40.48 & 7244.07 & 0.638 \\
$D^{*+} \bar{B}$ & $1 \mathrm{~S}$ & 40.48 & 7247.38 & 0.638 \\
\hline$D^{* 0} \bar{B}^{*}$ & $1 \mathrm{~S}$ & 42.63 & 7287.34 & 0.624 \\
$D^{*+} \bar{B}^{*}$ & $1 \mathrm{~S}$ & 42.63 & 7290.64 & 0.624 \\
\hline
\end{tabular}




\section{POTENTIAL FOR THE $(Q \bar{q})-(Q \bar{q})$ SYSTEM}

From our present model of effective charges, it is easy to see how the direct potential for the $(Q \bar{q})-(q \bar{Q})$ system depends on the ratio of the heavy quark mass $m_{Q}$ to the light quark mass $m_{q}$. If the mass of $Q$ is the same as the mass of $q$, then the attraction between effective charges of opposite signs will be compensated by the repulsive interaction between effective charges of the same sign, when we bring the two mesons together. The net direct potential is zero when $m_{Q}=m_{q}$. The polarization potential is always attractive, but the magnitude of the polarization potential in most cases is not attractive enough to lead to a bound meson-meson system.

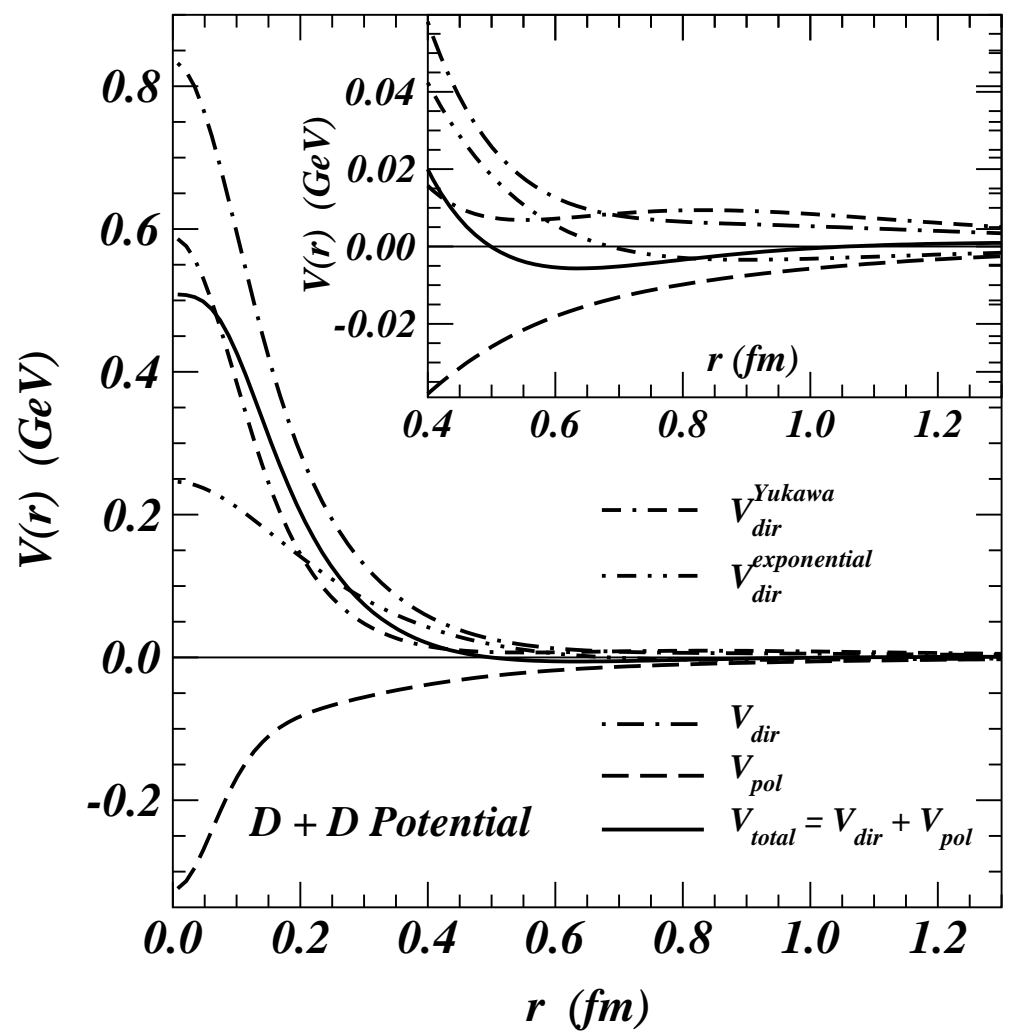

FIG. 7: The total potential $V(r)$ between $D$ and $D$ is shown as the solid curve. Shown also are various components of the potential. The insert gives the potentials at large separations in greater detail.

If $m_{Q} \gg m_{q}$, the light quark and antiquark in $(Q \bar{q})-(q \bar{Q})$ will move around a pair of nearly static heavy quark and heavy antiquark. As one brings a $(Q \bar{q})$ in the vicinity of a $(q \bar{Q})$ meson, the light quark and the light antiquark will attract each other because of their opposite effective charges and the meson-meson interaction will be attractive. The greater the mass ratio $m_{Q} / m_{q}$, the greater their attraction will be. Furthermore, the attractive polarization potential extends to large separations and facilitates the formation of molecular states.

From the above argument, one notes that the direct potential will be repulsive for the $(Q \bar{q})-(Q \bar{q})$ system which has two heavy quarks and two light antiquarks. In this case, as the mesons are brought close together, the two antiquarks moving around the nearly static heavy quarks will interact. As the effective charges of the antiquarks have the same sign, they tend to repel each other and the resultant direct interaction is repulsive. We show in Fig. 7 the total potential and its various components for the $D D$ system which has a repulsive direct potential. Although the polarization potential is attractive, the total potential is only very weakly attractive between $0.5 \mathrm{fm}$ and $1 \mathrm{fm}$ with a maximum depth of about $6 \mathrm{MeV}$. Such a shallow potential is not attractive enough to hold a molecular state. It should be pointed out, however, that symmetrization or anti-symmetrization of the spatial wave functions of the light antiquarks orbiting around the heavy quarks will give rise to additional effects [36, 37]. These effects must be studied in more detail for the $Q Q \bar{q} \bar{q}$ system, as in the case of electrons in a diatomic molecule. In these future studies, the direct potential and the polarization potential will provide some of the information needed for such an investigation. 


\section{CONCLUSION AND DISCUSSIONS}

We have studied four-quark $(Q \bar{q})-(q \bar{Q})$ systems involving charm and bottom quarks in a quark-based model. The system is described by a non-relativistic four-body Hamiltonian with a pairwise interaction.

We note that the molecular state of our interest is weakly-bound and the average separation $R$ between the heavy quark mesons is considerably greater than the average radius $a$ of the mesons. As $R>>a$, the dominant process for the interaction of the mesons is the exchange of two gluons, which leads to the color van der Waals interaction [53-55]. We can equivalently represent the color van der Waals interaction in terms of effective charges for quarks and antiquarks in a QED-type interaction. These effective charges give the proper description of bound states for a quark interacting with an antiquark in an isolated mesons. They also gives rise to a color-electric dipole-dipole interaction between mesons when a meson is brought in close proximity to another meson [53-55]. The color van der Waals interaction should be screened by introducing a screening mass $\mu$ due to the breaking of the gluonic string at large distance. Incorporating these concepts in a phenomenological interquark potential, we find a weakly-bound $D^{+} D^{*-}$ state near the threshold that may be qualitatively identified as the 3872 state observed recently by the Belle Collaboration.

In the present model, the meson-meson potential for the $(Q \bar{q})-(q \bar{Q})$ system can be decomposed into a direct potential and a polarization potential. The direct potential depends on the ratio of the quark masses $m_{Q} / m_{q}$. If the mass ratio is 1 , then the direct potential is zero. The magnitude of the attractive direct potential increases as the mass ratio increases. The polarization potential is always attractive. However, only when the quark mass ratio of both mesons are sufficiently high can the total potential be attractive enough to lead to bound states. There are no bound molecular states for $(s \bar{q})-(q \bar{s})$ and $(c \bar{q})-(q \bar{s})$ systems if we use meson-meson potentials obtained in the present model. We find bound meson-meson states in open heavy-quark systems involving combinations of $\left\{D, D^{*}, B, B^{*}\right\}$ with $\left\{\bar{D}, \bar{D}^{*}, \bar{B}, \bar{B}^{*}\right\}$. For open bottom meson pairs, we found weakly-bound $2 \mathrm{~S}$ states near the threshold, in addition to deeper $1 \mathrm{~S}$ states with binding energies of about $150 \mathrm{MeV}$.

We have included only screened color-Coulomb and screened linear confining interactions in the present work. It will be of interest to study in future work the influence of the spin-spin and other components of the interaction on the molecular structure. In meson systems having spin zero and one, the spin-spin interaction does not contribute to the direct potential when the spin of one of the mesons is zero. The effect of spin-spin interaction is expected to be small when one of the mesons is $D, B, \bar{D}$, or $\bar{B}$.

The present model differs from those of the pion-exchange models and other previous multi-quark models [39, 17-41, 45-49]. The results of molecular states obtained from different models can naturally be different. The present model predicts many molecular states in the combinations of $\left\{D, D^{*}, B, B^{*}\right\}$ with $\left\{\bar{D}, \bar{D}^{*}, \bar{B}, \bar{B}^{*}\right\}$. While it is encouraging that the present phenomenological model gives a molecular $D^{+} \bar{D}^{*-}$ state at about the right energy as the 3872 state, further careful comparisons of different predictions with experiment will be needed to determine whether the 3782 state is a molecular state. Close and Page [4] and Voloshin [6] suggest that if the 3872 state is a molecular state, its $D$ and $\bar{D}^{*}$ components should decay with a width equal to those of the isolated mesons, and the 3872 state should also be seen in the invariant mass of of the combination of the decay products of $D$ and $\bar{D}^{*}$. Furthermore, while the present model gives $D \bar{D}$ and $B \bar{B}$ molecular states, the pion-exchange model [3, 23] does not predict $D \bar{D}$ and $B \bar{B}$ molecular states. A search for $D \bar{D}$ and $B \bar{B}$ molecular states will be of interest to distinguish the different mechanisms that are present in generating the molecular states.

\section{Acknowledgments}

The authors would like to thank Profs. T. Barnes, K. F. Liu, and E. S. Swanson for helpful discussions. This research was supported in part by the Division of Nuclear Physics, U.S. Department of Energy, under Contract No. DE-AC05-00OR22725, managed by UT-Battelle, LLC.

[1] S.-K. Choi et al, the Belle Collaboration, Phys. Rev. Lett. 91, 262001 (2003); K. Abe et al., the Belle Collaboration, hep-ex/0308029; S.-K. Choi, et al, the Belle Collaboration, hep-ex/0309032.

[2] Talk presented by G. Bauer at the Second Heavy Quarkonium Workshop, Fermilab, September 20-22, 2003; D. Acosta et al, the CDFII Collaboration, Fermilab-Pub-03/393-E, hep-ex/0312021.

[3] N. A. Törnqvist, hep-ph/0308277.

[4] F. Close and P. R. Page, hep-ph/0309253.

[5] S. Pakavasa and M. Suzuki, Phys. Lett. B579, 74 (2004), hep-ph/0309294.

[6] M. B. Voloshin, hep-ph/0309307 
[7] P. Bicudo, G. M. Marques, hep-ph/0310008

[8] E. Eichten, talk presented at the Second Heavy Quarkonium Workshop, Fermilab, September 20-22, 2003.

[9] K. T. Chao, talk presented at the Second Heavy Quarkonium Workshop, Fermilab, September 20-22, 2003.

[10] E. Braaten and M. Kusunoki, hep-ph/0311147.

[11] E. S. Swanson, Physics Letters (in press), hep-ph/0311229.

[12] T. Barnes and S. Godfrey, Phys. Rev. D 69, 054008, hep-ph/0311162.

[13] C. Z. Yuan, X. H. Mo, P. Wang, Phys. Lett. B579, 74 (2004).

[14] E. Eichten, K. Gottfried, T. Kinoshita, K. D. Lane and T. M. Yan, Phys. Rev. D21, 203 (1980).

[15] W. Buchmüller and S-H.H. Tye, Phys. Rev. D24, 132 (1981).

[16] E. J. Eichten, K. Lane, and C. Quigg, Phys. Rev. Lett. 89, 162002 (2002).

[17] A. De Rujula, H. Georgi and S. L. Glashow, Phys. Rev. Lett. 38, 317 (1977); M.B. Voloshin and L.B. Okun, JETP Lett. 23, 333 (1976); M. Bander, G. Shaw, P. Thomas, and S. Meshkov, Phys. Rev. Lett. 36, 695 (1976).

[18] R. J. Jaffe, Phys. Rev. D15, 267 (1976), ibid D15, 281 (1976).

[19] A. D. Dolgov, L. B. Okun, and V. I. Zakharov, Phys. Lett. 49B, 455 (1974).

[20] K. F. Liu and C. W. Wong, Phys. Rev. D21, 1350 (1980).

[21] C. W. Wong and K. F. Liu, Phys. Rev. D21, 2039 (1980).

[22] J. Weinstein and N. Isgur, Phys. Rev. Lett. 48, 659 (1982); J. Weinstein and N. Isgur, Phys. Rev. D27, 588 (1983); J. Weinstein and N. Isgur, Phys. Rev. D41, 2236 (1990).

[23] N. A. Törnqvist, Phys. Rev. Lett. 67, 556 (1992); N. A. Törnqvist, Z. Phys. C61, 525 (1994).

[24] A. V. Manohar and M. B. Wise, Nucl. Phys. B 399, 17 (1993).

[25] T. E. O. Ericson and G. Karl, Phys. Lett. B309, 426 (1993).

[26] J. Weinstein and N. Isgur, Phys. Rev. D41, 2236 (1990).

[27] T. Barnes, Talk presented at the XXIX Recontres de Moriond, Meribel, France, 19-26 March 1994 hep-ph/9406215.

[28] T. Barnes, F. E. Close, and H. J. Lipkin, Phys. Rev. D68, 054006, 2003.

[29] K. Dooley, E. S. Swanson, T. Barnes, Phys. Lett. B275, 478 (1992).

[30] H. J. Lipkin, Phys. Lett. 70B, 113 (1977); H. J. Lipkin, Phys. Lett. 172B, 242 (1986); N. Isgur and H. J. Lipkin, Phys. Lett. 99B, 151 (1981).

[31] J. P. Ader, J.M. Richard, and P. Taxil, Phys. Rev. D25, 2370 (1982).

[32] L. Heller and J.A. Tjon, Phys. Rev. D32, 755 (1985).

[33] D. M. Brink and F. Stancu, Phys. Rev. D57, 6778 (1998).

[34] A. M. Green and P. Pennanen, Phys. Lett. B426, 243 (1998); A. M. Green and P. Pennanen, Phys. Rev. C57, 3384 (1998).

[35] A. Mihaly et al., Phys. Rev. D55, 3077 (1997).

[36] C. Michael and P. Pennanen, Phys. Rev. D60, 054012 (1999).

[37] T. Barnes, N. Black, D. J. Dean, and E. S. Swanson, Phys. Rev. C60, 045202 (1999).

[38] J.-M. Richard, hep-ph/0212224.

[39] S. Godfrey and N. Isgur Phys. Rev. D32, 189-231 (1985)

[40] T. Barnes and E. S. Swanson, Phys. Rev. D46, 131 (1992).

[41] E. S. Swanson, Ann. Phys. (N.Y.) 220, 73 (1992).

[42] C. Y. Wong, E. S. Swanson, and T. Barnes, Phys. Rev. C65, 014903 (2002).

[43] T. Barnes, E. S. Swanson, C. Y. Wong, and X.-M. Xu, Phys. Rev. C68, 014903 (2003).

[44] C. Y. Wong, E. S. Swanson, and T. Barnes, Phys. Rev. C C62, 045201 (2000).

[45] L. Ya. Glozman and D. O. Riska, Phys. Rep. 268, 263 (1996).

[46] Y. Fujiwara, C. Nakamoto, and Y. Suzuki, Phys. Rev. C54, 2180 (1996).

[47] L. Ya. Glozman, W. Plessas, K. Varga, and R. F. Wagenbrunn, Phys. Rev. D58, 094030 (1998).

[48] K. F. Liu et al., Phys. Rev. D59, 112001 (1999).

[49] Fl. Stancu and D. O. Riska, hep-ph/0307010.

[50] H. J. Lipkin, 113B, 490 (1982).

[51] O. W. Greenberg and H. J. Lipkin, Nucl. Phys. A370, 349 (1981).

[52] T. Appelquist, M. Dine, and I. J. Muzinich, Phys. Rev. D17, 2074 (1977).

[53] M. E. Peskin, Nucl. Phys. B156, 365 (1979).

[54] G. Bhanot and M. E. Peskin, Nucl. Phys. B156, 391 (1979).

[55] G. Bhanot, W. Fischler, and S. Rudaz, Nucl. Phys. B155, 208 (1979).

[56] E. Almqvist, D. A. Bromley, and J. A. Kuehner, Phys. Rev. Lett. 4, 515 (1960).

[57] Y. Kondo, Y. Abe, and T. Matsuse, Phys. Rev. C19, 1356 (1979).

[58] G. R. Satchler, Direct Nuclear Reactions, (Oxford University Press, Oxford, 1983); G. R. Satchler and W. G. Love, Phys. Rep. 55, 183 (1979).

[59] C. Y. Wong and H. W. Crater, Phys. Rev. C63, 044907 (2001).

[60] H. Lipkin, Phys. Lett. B45, 267 (1974); R. S. Willey, Phys. Rev. D18, 270 (1978); P. M. Fishbane and M. T. Grisaru, Phys. Lett. B74, 98 (1978); G. Feinberg and J. Sucher, Phys. Rev. D20, 1717 (1979); S. Matsuyama and H. Miyazawa, Prog. Theo. Phys. 61, 942 (1979); Y. Fujii and K. Mima, Phys. Lett. B79, 138 (1978).

[61] H. B. G. Casimir and D. Polder, Phys. Rev. 73, 360 (1947).

[62] J. Lucinda, J. Phys. A 24, 1759 (1991).

[63] G. S. Bali, Phys. Rev. D62, 114503 (2000)

[64] W. N. Zhang and C. Y. Wong, Phys. Rev. C68, 035211 (2003). 
[65] C. Y. Wong, Phys. Rev. D60, 114025 (1999).

[66] C. Y. Wong, Introduction to High-Energy Heavy-Ion Collisions, World Scientific Publishing Company, 1994.

[67] J. Schwinger, Phys. Rev. 82, 664 (1951).

[68] F. Petrovich, Nucl. Phys. A251, 143 (1975). 